\title{
Cylindrical two-dimensional electron gas in a transverse magnetic field
}

\author{
Giulio Ferrari, ${ }^{1, *}$ Andrea Bertoni, ${ }^{1}$ Guido Goldoni, ${ }^{1,2}$ and Elisa Molinari ${ }^{1,2}$ \\ ${ }^{1}$ CNR-INFM Research Center on nanoStructures and bioSystems at Surfaces (S3), Via Campi 213/A, 41100 Modena, Italy \\ ${ }^{2}$ Dipartimento di Fisica, Università di Modena e Reggio Emilia, Via Campi 213/A, 41100 Modena, Italy \\ (Received 19 June 2008; revised manuscript received 3 September 2008; published 30 September 2008)
}

\begin{abstract}
We compute the single-particle states of a two-dimensional (2D) electron gas confined to the surface of a cylinder immersed in a magnetic field. The envelope-function equation is solved exactly for both a homogeneous and a periodically modulated magnetic field perpendicular to the cylinder axis. The nature and energy dispersion of the quantum states reflects the interplay between different length scales, namely, the cylinder diameter, the magnetic length, and, possibly, the wavelength of the field modulation. We show that a transverse homogeneous magnetic field drives carrier states from a quasi-2D (cylindrical) regime to a quasi-onedimensional regime where carriers form channels along the cylinder surface. Furthermore, a magnetic field which is periodically modulated along the cylinder axis may confine the carriers to tunnel-coupled stripes, rings, and dots on the cylinder surface depending on the ratio between the field periodicity and the cylinder radius. Results in different regimes are traced to either incipient Landau-level formation or Aharonov-Bohm behavior.
\end{abstract}

DOI: 10.1103/PhysRevB.78.115326

PACS number(s): 73.20.At, 73.22.-f, 73.21.-b

\section{INTRODUCTION}

The interest in the electronic properties of quantum systems with cylindrical symmetry has received a boost since the early proposals of adopting carbon nanotubes ${ }^{1,2}$ as building blocks for future nanoelectronic devices, exploiting their peculiar mechanical and electrical properties. ${ }^{3,4}$ In recent years new inorganic semiconductor systems are also emerging where carriers are confined on a bent surface, and several possibilities arise to obtain two-dimensional (2D) electron gases (2DEGs) with cylindrical symmetry (C2DEGs), which may enrich the wealth of physics and applications of planar semiconductor nanostructures. One such system can be obtained from a standard epitaxially grown 2DEG at a planar heterojunction, which is then overgrown with a latticemismatched material at some distance above the buried 2DEG. A sacrificial layer below the 2DEG allows the release of the elastic energy by liftoff and bending of a thin layer of material embedding the $2 \mathrm{DEG}$ up to complete rolling. ${ }^{5-8}$ The rolled-up layers stick together, thus forming a C2DEG with a radius ranging from tens of nanometers up to several microns, showing peculiar magnetoresistance with respect to the corresponding planar structures. ${ }^{8}$ Alternatively, a C2DEG can be obtained in coaxial structures which can be fabricated similarly to standard layered heterostructures, but using a cylindrical substrate rather than the usual planar substrate for multilayer overgrowth of lattice matched materials. The cylindrical substrate, in turn, can be obtained by a self-standing single-crystal semiconductor nanowire, fabricated by seeded growth, either assisted by Au (Refs. 9 and 10) or Ga (Ref. 11) nanoparticles. The latter possibility is particularly promising for high-mobility nanodevices to avoid Au-induced deep level traps. The resulting C2DEGs will have a diameter determined by the diameter of the nanowire used as a substrate in the few tens of nanometer range.

Although carbon nanotubes and C2DEGs share the cylindrical symmetry of the electronic states, they have very different curvatures. Carbon nanotubes have typical diameters in the few nanometers range and are basically quasi-onedimensional (1D) systems, while the diameter of C2DEGs is at least one order of magnitude larger. For this reason, we expect the electronic properties of the latter systems to be dominated by size quantization, as in usual planar heterostructures, rather than by the atomistic details. In addition, the effect of an external magnetic field is stronger in C2DEGs than in carbon nanotubes since the magnetic length of typical fields is comparable to the lateral dimension, while carbon nanotube diameters are much smaller. Therefore, the interplay between the cylinder diameter and the magnetic length adds a new degree of freedom to manipulate the quantum states of the carriers.

It can also be noticed that the field itself may be modulated on the scale of the nanostructure diameter. ${ }^{12,13}$ In fact, modulated fields can be obtained by means of ferromagnetic strips or dots on top of a $2 \mathrm{DEG}$, which give rise to effective periodic potentials and hence to oscillatory behaviors in the magnetoresistance of planar structures. ${ }^{14,15}$ These oscillations in the magnetoresistivity and their effect on the electronic properties of planar 2DEGs have been studied extensively. ${ }^{16-18}$ This is the counterpart of homogeneous magnetic fields applied to modulated periodic 2D structures, which may induce complex and fascinating electronic properties such as the Hofstadter butterfly. ${ }^{19,20}$ It is therefore interesting to couple modulated magnetic fields with structures having a built-in periodicity of the carrier states on the same length scale such as the C2DEGs introduced above.

In this paper, we investigate the effect of a transverse magnetic field on the single-particle properties of carriers in a C2DEG. Electronic band structures and density of states (DOS) are obtained with the magnetic field either uniform or periodically modulated along the cylinder. In the former case, the field does not break the translational invariance along the axis of the cylinder and the electronic states scale with the ratio between the cylinder diameter and the magnetic length. The system is found to show a transition from a quasi-2D to a quasi-1D behavior as a function of the field strength. On the contrary, a modulated magnetic field breaks 
the continuous translational symmetry of the cylindrical 2DEG leading to the formation of energy subbands and opens gaps along the cylinder axis. In such system the effect of the field depends on the interplay between its intensity (or magnetic length), the radius of the tube, and the wavelength of the field modulation. We find that the tailoring of different length scales may lead to the formation of electronic states similar to those observed in arrays of antidots, ${ }^{21}$ with the magnetic field giving rise to a series of regions where the carriers cannot penetrate, if not belonging to very high energy bands. Allowed regions form a network of tunnelcoupled dots, rings, and arrays depending on the ratio between the cylinder radius and the field periodicity.

The paper is organized as follows. In Sec. II, the cylindrical system under study is introduced and its Schrödinger equation is derived. In Sec. III, the theory is applied to the case of a homogeneous transverse field and different regimes of 1D localization are discussed. Section IV addresses the effect of a spatially modulated magnetic field in different regimes of tube radius, field modulation wavelength, and field strength. We also discuss our results in connection with Aharonov-Bohm effects and Landau-level formation. In Sec. $\mathrm{V}$, the conclusions are drawn.

\section{HAMILTONIAN OF A C2DEG IN A MAGNETIC FIELD}

We consider the problem of a spinless electron bound to the surface $S$ of a cylinder in a magnetic field perpendicular to its axis. The general derivation of a proper quantum equation of motion on a curved surface is a problem with a long history. The classical approaches are the Lagrangian method of de Witt ${ }^{22}$ and the limiting procedure of Jensen-Koppe ${ }^{23}$ and da Costa. ${ }^{24}$ While the former includes the effects of the curvature directly in the equation of motion, the latter is most appropriate when the system under consideration is a real surface embedded in the three-dimensional (3D) space $^{25-28}$ as in our case. When a magnetic field is introduced, the problem gets more complicated, but experimental measures pointed out more than a decade ago that the transport properties of nonplanar 2DEGs in a homogeneous magnetic field show an oscillatory behavior due to the nonuniform component of the field perpendicular to the $2 \mathrm{DEG} .{ }^{29}$ The same type of measures has been recently repeated on C2DEGs, ${ }^{8,30,31}$ showing a dependence of the magnetoresistance on the intensity and direction of the magnetic field. From a theoretical point of view, only recently it has been shown, by one of the authors, ${ }^{32}$ that an analytical expression of the Schrödinger equation where the dynamics on the surface is decoupled from the transverse one can always be obtained provided that a proper choice of the gauge is made. However, for the present case of a straight cylinder with constant radius $r$ the solution is not difficult and can be achieved using also different approaches. ${ }^{27,33-35}$

Let the cylinder axis be along the $y$ direction of a Cartesian reference system with $x$ and $z$ perpendicular to $S$ (see Fig. 1). We also define two coordinates $q_{1}$ and $q_{2}$ lying on $S$ and $q_{3}$ perpendicular to $S$ as shown in Fig. 1. Therefore, $S$ is defined by
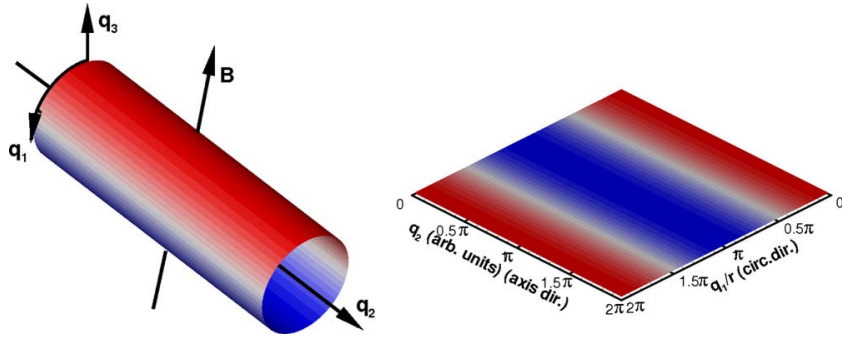

FIG. 1. (Color online) The cylindrical surface $S$ in a homogeneous magnetic field $B$. The cylindrical reference frame used throughout the paper is indicated. The intensity of the field component normal to $S$ is shown in color code both in space and as an open surface with darker colors indicating a stronger field.

$$
\left\{\begin{array} { l } 
{ x = r \operatorname { s i n } ( q _ { 1 } / r ) , } \\
{ y = q _ { 2 } , } \\
{ z = r \operatorname { c o s } ( q _ { 1 } / r ) , }
\end{array} \text { with } \quad \left\{\begin{array}{l}
q_{1} \in[0 ; 2 \pi), \\
q_{2} \in(-\infty ; \infty), \\
q_{3}=0 .
\end{array}\right.\right.
$$

Next, we consider a magnetic field perpendicular to the cylinder axis. For definiteness, we set the magnetic field along the $z$ axis, $\vec{B}=[0 ; 0 ; B(y)]$, with an intensity $B(y)$ possibly modulated along the $y$ direction. A convenient choice for the vector potential is $\vec{A}=[0 ; x B(y) ; 0]$, which in the cylindrical frame is

$$
\vec{A}\left(q_{1}, q_{2}\right)=\left(A_{1} ; A_{2} ; A_{3}\right)=\left[0 ; r B\left(q_{2}\right) \sin \left(q_{1} / r\right) ; 0\right] .
$$

With this choice of the gauge, the Hamiltonian reads ${ }^{32}$

$$
\begin{aligned}
H= & -\frac{\hbar^{2}}{2 m}\left(\frac{\partial^{2}}{\partial q_{1}^{2}}+\frac{\partial^{2}}{\partial q_{2}^{2}}\right)+\frac{i e \hbar}{2 m}\left(\frac{\partial}{\partial q_{2}} A_{2}+A_{2} \frac{\partial}{\partial q_{2}}\right) \\
& +\frac{1}{2 m} e^{2} A_{2}^{2}-\frac{\hbar^{2}}{8 m r^{2}},
\end{aligned}
$$

where $e>0$ is the elementary charge and $m$ is the electron mass, respectively. The last term in Eq. (3) is the potential arising from the (constant) curvature of the surface ${ }^{24}$ and will be dropped in the following since it amounts to a rigid energy shift. Note that, for this particular surface and field configuration, the above equation can also be obtained by writing the Laplacian term contained in the Hamiltonian in cylindrical coordinates using the Peierls substitution ${ }^{36}$ based on the principle of minimal coupling $i \hbar \frac{\partial}{\partial \vec{q}} \rightarrow\left(i \hbar \frac{\partial}{\partial \vec{q}}+e \vec{A}\right)$.

\section{HOMOGENEOUS MAGNETIC FIELD}

We next apply the general formalism to the case of a cylinder in a homogeneous magnetic field of intensity $B$. Here, the vector potential in Eq. (2) reads $\vec{A}\left(q_{1}\right)$ $=\left[0 ; r B \sin \left(q_{1} / r\right) ; 0\right]$. The geometry is shown in Fig. 1, where the direction of the field is represented by the upward arrow, while the intensity of its component normal to $S$ is indicated by the light (low value) and dark (high value) colors.

The vector potential depends only on $q_{1}$, i.e., the position along the circumference of the cylinder and does not break the translational invariance along $q_{2}$. Since the field does not 


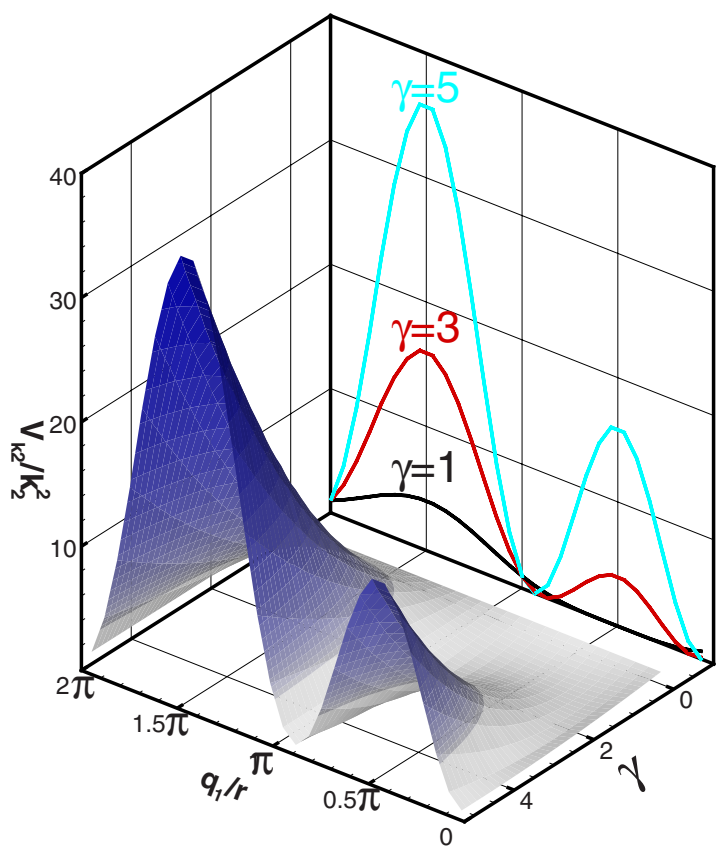

FIG. 2. (Color online) The effective potential $V_{k_{2}}\left(q_{1}\right) / k_{2}^{2}$ as a function of the position around the circumference $q_{1}$ and of the coupling parameter $\gamma$. For low $\gamma, V_{k_{2}}\left(q_{1}\right)$ has one shallow minimum at $q_{1}=(\pi / 2) r$, which bifurcates into two deeper minima at larger values of $\gamma$. At back of the graph, the potential is shown at three selected values of $\gamma$ as indicated.

depend on $q_{2}$, the wave function separates as

$$
\Psi\left(q_{1}, q_{2}\right)=\varphi\left(q_{1}\right) e^{i k_{2} q_{2}},
$$

where $k_{2}$ is the wave vector along the axis. The Hamiltonian reads

$$
\mathbb{H}=\frac{\hbar^{2}}{2 m}\left[-\frac{\partial^{2}}{\partial q_{1}^{2}}+V_{k_{2}}\left(q_{1}\right)\right],
$$

where

$$
V_{k_{2}}\left(q_{1}\right)=k_{2}^{2}\left(1-\frac{e B r}{\hbar k_{2}} \sin \frac{q_{1}}{r}\right)^{2}
$$

is a wave-vector-dependent $1 \mathrm{D}$ effective potential that wraps around the circumference of the cylinder. Figure 2 shows the profile of $V_{k_{2}}\left(q_{1}\right) / k_{2}^{2}$, as a function of the dimensionless parameter

$$
\gamma=\frac{e B r}{\hbar k_{2}}
$$

that gives the strength of the interaction between the charge and the field, for a given wave vector value. For $\gamma \leq 1$ the effective potential has one shallow minimum at $q_{1}=(\pi / 2) r$. As a consequence the carriers tend to localize in a quasi-1D channel on one side of the cylinder, where the component of the field normal to $S$ is minimum, which side being decided by the relative sign of the wave vector and the field. For $\gamma$ $\gtrsim 1, V_{k_{2}}\left(q_{1}\right) / k_{2}^{2}$ has two minima which, for large $\gamma$, are located at $q_{1}=0$ and $q_{1}=\pi r$, i.e., above and below the cylinder. In this regime, carriers form two quasi-1D channels located where the component of the field normal to $S$ is maximum, and the field is either entering or exiting the surface.

\section{A. Energy levels}

For a given $k_{2}$, we found the exact eigenstates of the Hamiltonian $\mathbb{H}$ by writing the $\varphi\left(q_{1}\right)$ component of $\Psi$ as a linear combination of the modes on the circumference

$$
\varphi\left(q_{1}\right)=\frac{1}{\sqrt{2 \pi}} \sum_{n} c_{n} e^{i \frac{n}{r} q_{1}} .
$$

The zero-field energies of the 2D states are

$$
\epsilon_{n}\left(k_{2}\right)=\frac{\hbar^{2}}{2 m}\left(\frac{n^{2}}{r^{2}}+k_{2}^{2}\right),
$$

where $n$ is an integer labeling the modes on the circumference mixed by the magnetic field. By direct diagonalization of Eq. (5) on the basis of the modes on the circumference, we obtain the energies $\epsilon_{n}\left(k_{2}\right)$ and the eigenfunctions for any value of $B$. All reported calculations are obtained with $n$ $=20$ in Eq. (8). With such choice, the convergence is accurate within $1 \%$.

In order to discuss the eigenstates of a carrier in the presence of a homogeneous field, it is convenient to define the dimensionless coupling parameter

$$
\alpha^{c}=r \sqrt{\frac{2 \pi e B}{\hbar}},
$$

namely, the ratio between half of the circumference and the magnetic length calculated by averaging the intensity of the field over one half of the circumference. Clearly, this parameter describes the coupling between the field and the carrier. Apart from the averaging, it corresponds to the one defined in Ref. 33. We stress that the energies of the eigenstates scale with $\alpha^{c}$, i.e., the same energy is obtained for different values of the tube radius and field intensity as long as $r \sqrt{B}$ is constant.

The subband structure for a homogeneous field is shown in Fig. 3. At $\alpha^{c}=0$ (zero field), the energy bands are given by Eq. (9); therefore, all bands are doubly degenerate except for the lowest one. When a magnetic field is applied, the double degeneracy is lifted by the orbital Zeeman splitting. For high values of $\alpha^{c}$, that is, for sufficiently strong field at fixed tube radius, the subbands flatten at small $k_{2}$. By increasing $\alpha^{c}$, the energy becomes almost independent of the wave vector in a larger range and approaches the value of Landau levels in a planar 2D system. As we will see in the following these states correspond to Landau-type states confined above and below the cylinder.

\section{B. Density of states and magnetic-induced localization}

With the energy bands just calculated, it is possible to obtain the DOS for any given value of $\alpha^{c}$, applying the following formula: ${ }^{37}$ 

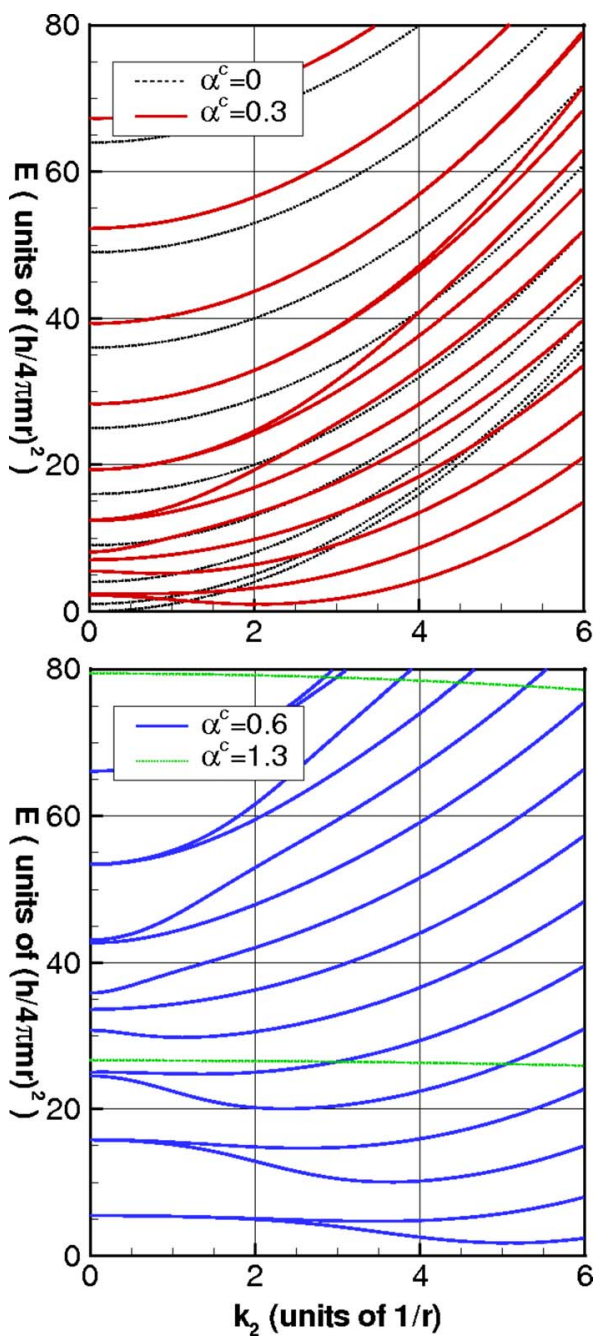

FIG. 3. (Color online) Energy subbands at selected values of the coupling parameter $\alpha^{c}$. The parabolic subbands $\alpha^{c}=0$ (zero field) are doubly degenerate except for the lowest one.

$$
\operatorname{DOS}(\epsilon)=2 \sum_{n, k_{2}} \delta\left[\epsilon-\epsilon_{n}\left(k_{2}\right)\right]
$$

where $\delta$ is a Dirac delta and the factor 2 is included for spin degeneracy. The DOS, as a function of the energy and $\alpha^{c}$, is shown in Fig. 4. We can recognize different regimes: (i) at small $\alpha^{c}$ (low field), the orbital Zeeman splitting lifts the double degeneracy of the zero-field bands; (ii) at intermediate fields, the energy of the states is lowered by the interaction with the field; and (iii) at large fields, different subbands merge into highly degenerate levels reminiscent of the corresponding 2D Landau level. In Fig. 4, the energy splitting due to the orbital Zeeman effect is shown for the two states $n=1$ (dash-dotted line) and $n=-1$ (solid line) ${ }^{48}$ for a specific $k_{2}$ value. As the field is switched on, the degenerate levels split, then the energy decreases and reaches a minimum for a value of $\alpha^{c}$ that is larger for larger $n$ and $k_{2}$. The locus of the minima of all the states with the same $n$ (and different wave vectors) gives a peak in the DOS, indicating the formation of a 1D state. In the top panel of Fig. 5 we show the DOS corresponding to $\alpha^{c}=1.3$. Peak A belongs to the locus of the

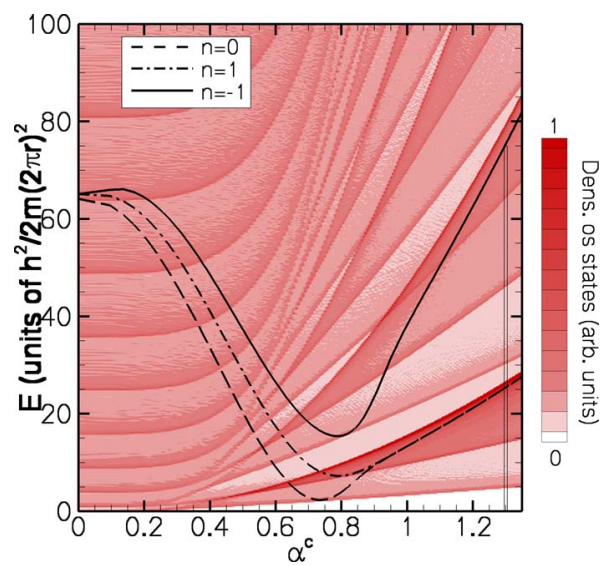

FIG. 4. (Color online) DOS as a function of the energy and the coupling parameter $\alpha^{c}$ (color code given in the legend). For reference, we show three energy levels corresponding to $k_{2} r=8$, which evolve from the $n=0, \pm 1$ levels at $\alpha^{c}=0$ as indicated. The vertical line at $\alpha^{c}=1.3$ shows the position of the DOS reported in Fig. 5 .

minima of the $n=0$ states and is strongly suggestive of the $\epsilon^{-1 / 2}$ behavior of a quasi-1D system, with $\epsilon$ being the energy with respect to the band edge. Analogously, peak B belongs to the locus of minima of the $n=1$ states. In the bottom panel of Fig. 5, a polar plot shows the probability densities corresponding to peaks $\mathrm{A}, \mathrm{B}$, and $\mathrm{C}$ (described later). These 1D states localize in the region of the cylinder where the field is parallel to the surface. In fact, they are edge states driven on one side of the cylinder by Lorentz force and this side is determined by the sign of the charge and by the direction of $B$. A change in the relative sign of these parameters switches the localization to the opposite side. In this regime the potential $V\left(q_{1}\right)$ has one minimum as shown in Fig. 2.

For high values of $\alpha^{c}$, the energy levels gather into Landau levels whose energy grows linearly with $B$. The first Landau level is formed, independently of $k_{2}$, by the states $n=0$ and 1 , the second one by the states $n=-1$ and 2 , and so on. The Landau levels are well recognizable in the density of states of Fig. 4 where they appear as dark lines. In the top panel of Fig. 5, the most prominent feature is peak $\mathrm{C}$ which corresponds to the formation of the first Landau level. Due to the finite curvature the energy levels acquire a finite dispersion, which gives rise to the 1D-like tail of the DOS on the low-energy side unlike the standard 2D Landau levels. The probability density of a Landau state is localized in the regions where the magnetic field is perpendicular to $S$; as shown in the bottom panel of Fig. 5 it has two lobes aligned with the magnetic field. In this case the region of localization is independent of the charge sign and of the direction of the field. The 1D Landau states are always the strips that run on the top and on the bottom of the cylinder. However, since this effect is related to the formation of Landau levels on the cylinder, the strength of the localization reaches a saturation for high $\alpha^{c}$. This means that the maximum localization in the circumferential direction is independent of the cylinder radius, of the intensity of the magnetic field, and of the wave vector $k_{2}$.

The detailed analysis given for the first three peaks of the DOS can be repeated for all other peaks. In general, as the 

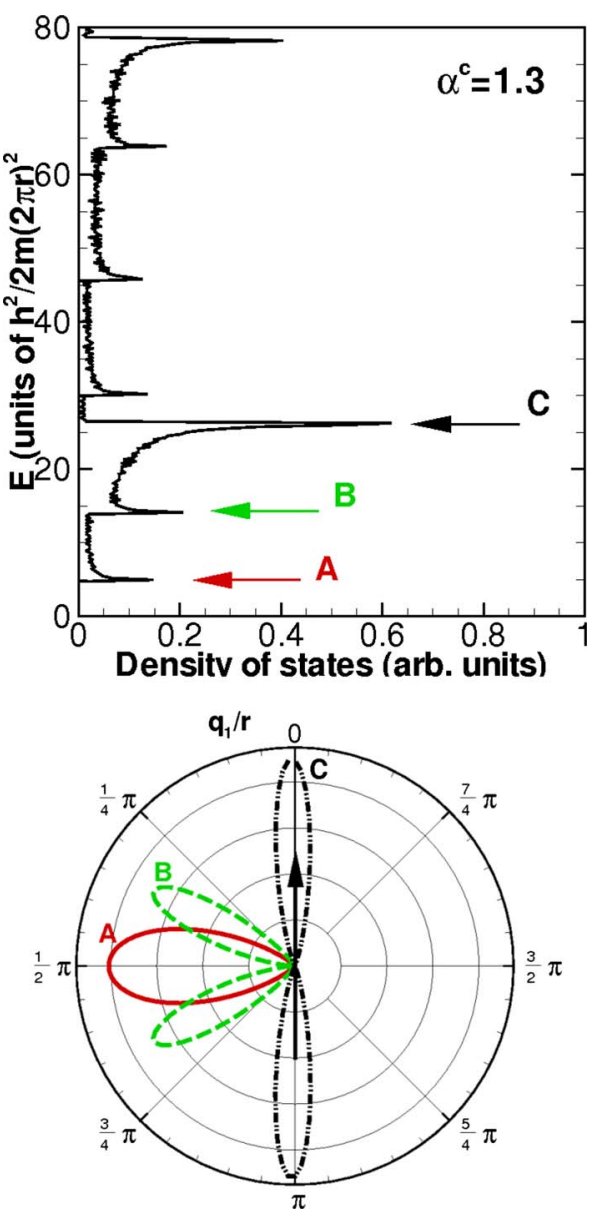

FIG. 5. (Color online) Top: DOS at $\alpha^{c}=1.3$. The presence of many peaks indicating new $1 \mathrm{D}$ states is clearly visible. The states of peaks A and B correspond to states with $n=0$ and 1 at zero field, respectively. Peak $\mathrm{C}$ contains states starting from both $n=0$ and 1 . For the given value of $\alpha^{c}$ they form 1D Landau levels. Bottom: Probability densities of the states corresponding to the peaks in the top panel. States A and B are 1D states localized in the region of the cylinder where $B$ is parallel to the surface: these are edge states driven in that position by Lorentz force. State $C$ belongs to a Landau level: these $1 \mathrm{D}$ states are localized in the regions where $B$ is perpendicular to the surface. The direction of the field is indicated by the arrow.

value of $\alpha^{c}$ increases, each state evolves in a 1D state of type $\mathrm{A}$ or $\mathrm{B}$ and then shrinks in a $1 \mathrm{D}$ Landau state.

\section{SPATIALLY MODULATED MAGNETIC FIELD}

We next consider the case of a magnetic field periodically modulated in intensity along the axis of the cylinder with zero average intensity. To be specific, we consider a magnetic field whose intensity varies with a sinusoidal law along the axis. The vector potential in Eq. (2) reads

$$
\vec{A}\left(q_{1}, q_{2}\right)=\left[0 ; r B \cos \left(q_{2} / R\right) \sin \left(q_{1} / r\right) ; 0\right],
$$

where $2 \pi R$ is the wavelength of the spatial modulation of the field along $q_{2}$ and $B$ is its maximum intensity. Also in the modulated-field case, we choose the gauge according to Eq.

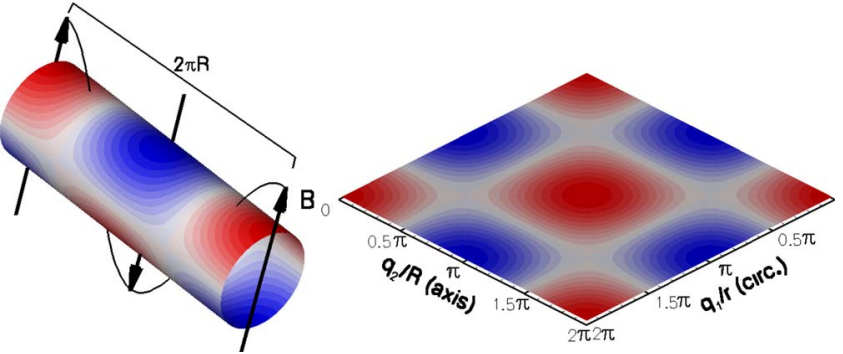

FIG. 6. (Color online) The cylindrical surface $S$ of radius $r$ in a magnetic field periodically modulated with wavelength $2 \pi R$ along the tube axis. The intensity of the field component normal to $S$ is shown in color code both in 3D space and on the open surface as in Fig. 1. Lighter colors indicate regions where the vertical component of the field is zero, either because the field is parallel to $S$ or because the intensity is zero.

(2) with $A_{3}=0$ that leads to the most suitable expression of the Hamiltonian. ${ }^{32}$ In Fig. 6, the arrows indicate the direction of the modulated magnetic field, while the intensity of its component perpendicular to $S$ is shown in color code. Note the square pattern formed by the white regions where the perpendicular component of the field vanishes, either because the field is parallel to $S$ or because it has a vanishing intensity there.

Since the vector potential now depends on both $q_{1}$ and $q_{2}$, the wave function cannot be separated as in Eq. (4), and we have to solve a fully 2D problem. However, since the Hamiltonian is periodic in $q_{2}$, we can apply the Bloch's theorem to a periodic system with a $1 \mathrm{D}$ unit cell of length $2 \pi R$ along $q_{2}$. The exact eigenstates $\chi\left(q_{1}, q_{2}\right)$ are found as a linear combination of the zero-field states as

$$
\chi\left(q_{1}, q_{2}\right)=\frac{1}{2 \pi} \sum_{n l} c_{n l}\left(k_{2}\right) e^{i \frac{n}{r} q_{1}} e^{i\left(k_{2}+l G\right) q_{2}},
$$

where $G=1 / R$ and $-G / 2 \leq k_{2} \leq G / 2$. The corresponding zero-field energies are

$$
\epsilon_{n l}\left(k_{2}\right)=\frac{\hbar^{2}}{2 m}\left(\frac{n^{2}}{r^{2}}+\frac{l^{2}}{R^{2}}+k_{2}^{2}\right) .
$$

Here, the quantum numbers $n$ and $l$ are integers representing the mode numbers around the circumference and along $q_{2}$ in the $1 \mathrm{D}$ unit cell, respectively. When a finite field is applied, the $n$ and $l$ modes are mixed. In this section, the results are obtained by taking $n=l=30$ in Eq. (13).

One useful parameter to characterize the system is $\alpha^{v}$ $=\Phi / \Phi_{0}$, which is the ratio between $\Phi$, namely, the magnetic flux through a cell $(\pi r \times \pi R)$ and the magnetic-flux quantum $\Phi_{0}=2 \pi \hbar / e$. Note that the field is not constant within the cell $(\pi r \times \pi R)$. Therefore, we have

$$
\Phi=\frac{1}{\pi^{2} r R} \int_{0}^{\pi r} \int_{0}^{\pi R} B\left(q_{1}, q_{2}\right) d q_{1} d q_{2}=4 B R r
$$

and 

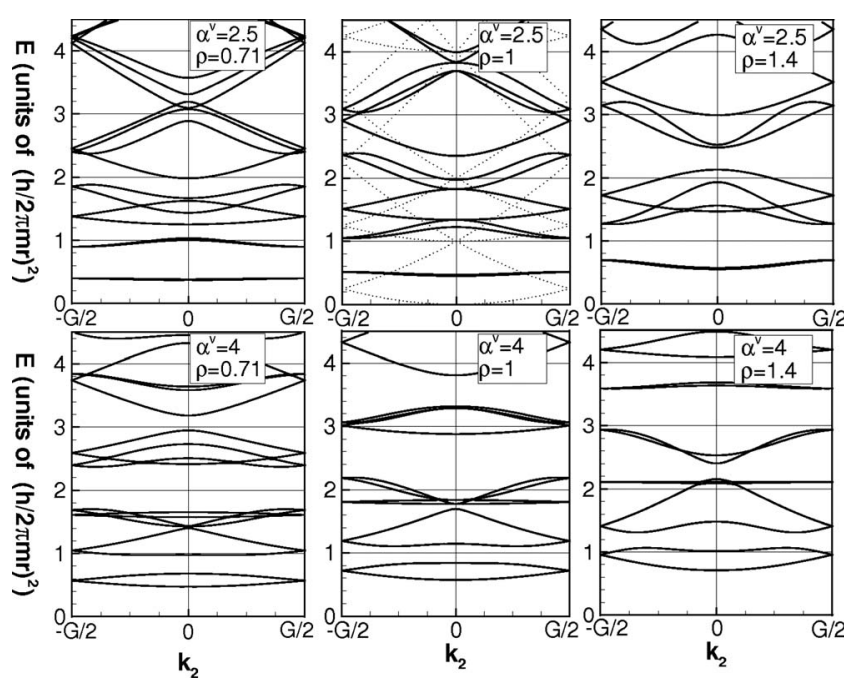

FIG. 7. Energy subbands for the three selected values of $\rho$ $=0.71,1,1.4$ (left, center, and right column) each at $\alpha^{v}=2.5,4$ (upper and lower row). In the upper central panel the parabolic subbands at zero field are also shown with dotted lines for comparison.

$$
\alpha^{v}=\frac{2 e B R r}{\pi \hbar} .
$$

The parameter $\alpha^{v}$ gives the strength of the coupling between the field and the carriers and plays a role analogous to $\alpha^{c}$ in the homogeneous case of Sec. III. The coupling increases linearly with the intensity of $B$ and with the spatial periodicities $r$ and $R$. Indeed, $\alpha^{v}$ is defined in analogy to other works involving magnetic field applied to a 2D system. ${ }^{19,38-41}$ In Ref. 19, $\alpha^{v}$ is alternatively interpreted as the ratio between the period of motion of an electron with crystal momentum $\hbar / \sqrt{r R}$ (which is $2 \pi r R m / \hbar$ ) and the reciprocal of the average cyclotron frequency $4 e B / \pi^{2} m$.

In general terms, the vector potential creates an effective 2D potential on the surface $S$ that tends to localize the wave function where the component of the field orthogonal to $S$ is zero (lighter regions in Fig. 6). Specifically, the low-energy states of the carriers will be mainly localized at the intersections of the above stripes. These quasi-zero-dimensional (0D) regions are connected by tunneling in a $2 \mathrm{D}$ network. The ratio $\rho=r / R$ between the cylinder radius $r$ and the length of the field modulation $R$ identifies different regimes, whether $\rho<1, \rho \approx 1$, or $\rho>1$ as we show next.

\section{A. Ringlike localization}

Let us start from the case $\rho<1$, namely, the field is slowly modulated with respect to the cylinder diameter and $r<R$. All the results of this subsection are for $\rho=0.71$. The left panels of Fig. 7 show the energy bands at two values of $\alpha^{v}$. Compared to the gapless parabolic band structure at zero magnetic field reported in the central upper panel (dotted lines), the finite-field band structure is characterized by large gaps in the low-energy range. Furthermore, the lowest subbands are almost flat for the values of $\alpha^{v}$ shown here, which indicates carrier localization.

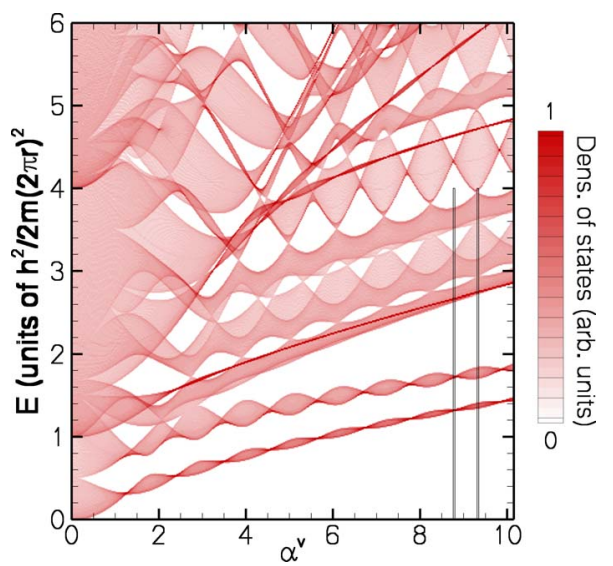

FIG. 8. (Color online) DOS at $\rho=0.71$ (see Sec. IV A) as a function of the energy and the coupling parameter $\alpha^{v}$ (color code given in the legend). The DOS shown in Fig. 9 corresponds to the two vertical lines at $\alpha^{v}=8.77$ and 9.33.

In Fig. 8 we show the DOS as a function of the coupling parameter $\alpha^{v}$. This clearly shows the opening of many energy gaps with an amplitude that strongly depends on $\alpha^{v}$. At somewhat regular values, the two lowest subbands are completely flat. The modulation in the DOS and the occurrence of peaks (darker lines in Fig. 8) is also shown in the top panel of Fig. 9 for two specific values of $\alpha^{v}$.

In the bottom panel of Fig. 9 we show the probability density on the tube for a state belonging to the peak in the top panel indicated with the arrow. The charge density is mainly distributed in a superlattice of quasi-0D states induced by the magnetic field localized at the intersections of the regions where the perpendicular component of $B$ is zero (see Fig. 6). The localization is stronger for increasing $\alpha^{v}$. For the present case of $\rho<1$, the asymmetric shape of the unit cell $(\pi r \times \pi R)$ turns into an asymmetric tunneling between the quasi-0D regions, where charge is mainly localized. At this value of $\alpha^{v}$, tunneling is almost suppressed along the axis direction, but it is present around the circumference. The effect is that the 2DEG is rearranged in arrays of weakly coupled pairs of quantum dots. Also, note that the positions of the dots along the axis are independent of the sign of the charges and, since the confinement occurs in the zeros of the effective field, the positions are also independent of the direction of the field. When $\rho$ is significantly smaller than 1 , the tunneling in the axial direction is completely suppressed and the particle probability is homogeneous in the circumference direction, so that in each unit cell two rings form at the positions $q_{2}=\frac{1}{2} \pi R$ and $\frac{3}{2} \pi R$.

\section{B. Striplike localization}

Next we analyze the case $\rho>1$, namely, the field modulation is rapid with respect to the tube diameter and $R>r$. All numerical results presented in this subsection are for $\rho$ $=1.40$. The energy subbands are shown in Fig. 7 (right panels) for two values of $\alpha^{v}$. Again, the magnetic field affects the dispersion and opens energy gaps. Almost flat bands can be observed for specific values of the $\alpha^{v}$ parameter and lo- 

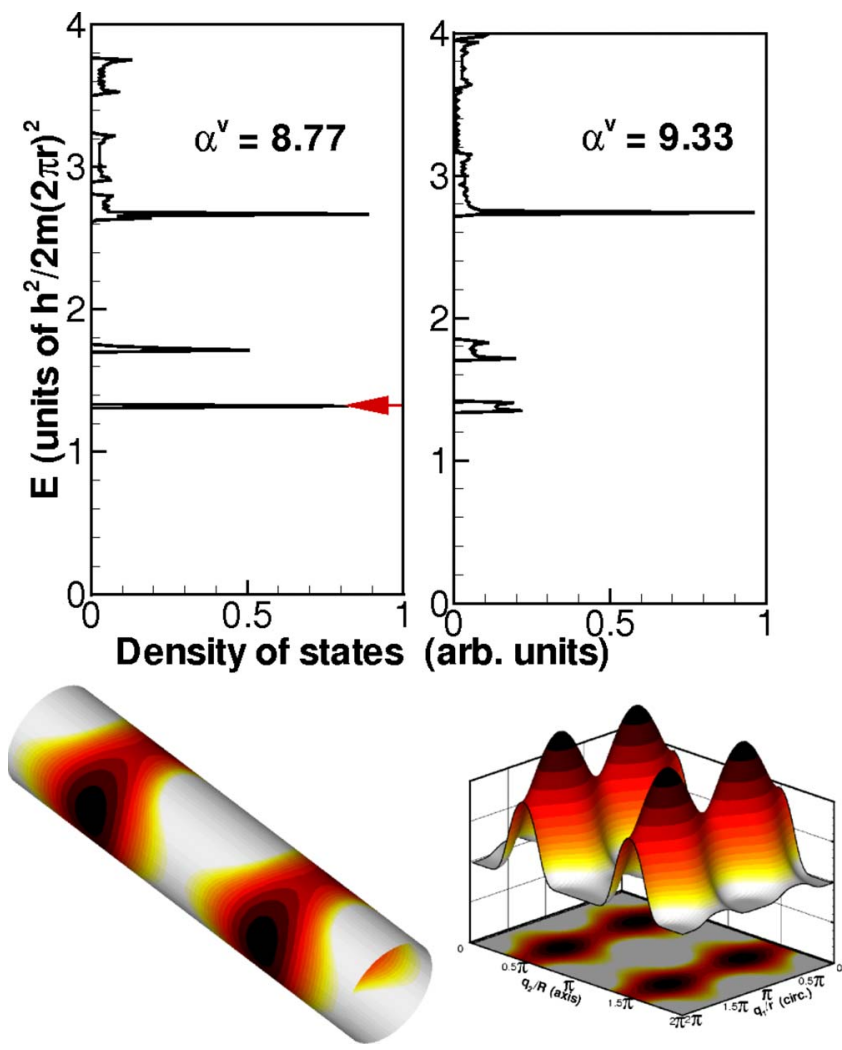

FIG. 9. (Color online) Top: DOS at $\rho=0.71$ for $\alpha^{v}=8.77$ and 9.33. Bottom: Probability density for a state belonging to the peak indicated with the arrow in the top panel. Darker color means higher density. The applied field rearranges the C2DEG in a lattice of quasi-0D states connected in a ringlike shape around the tube and weakly coupled along the tube.

calized states will be observed on the surface of the cylinder.

The DOS as a function of the parameter $\alpha^{v}$ is shown in Fig. 10 and in the top panel of Fig. 11. Again, the magnetic field has the main effect of opening gaps not present at zero field, although the gap pattern is different from Fig. 8.

In the bottom panel of Fig. 11, we show the probability density on the tube for a state belonging to the peak indicated

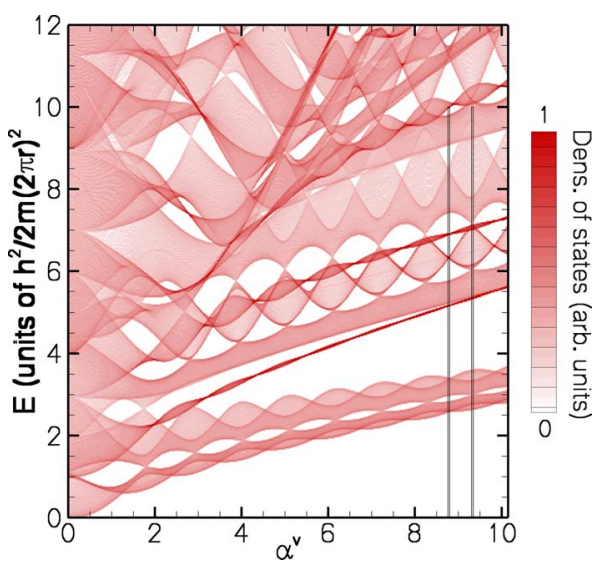

FIG. 10. (Color online) DOS at $\rho=1.40$ (see Sec. IV B) as a function of the energy and the coupling parameter $\alpha^{v}$ (color code given in the legend). The DOS shown in Fig. 11 corresponds to the two vertical lines at $\alpha^{v}=8.77$ and 9.33.
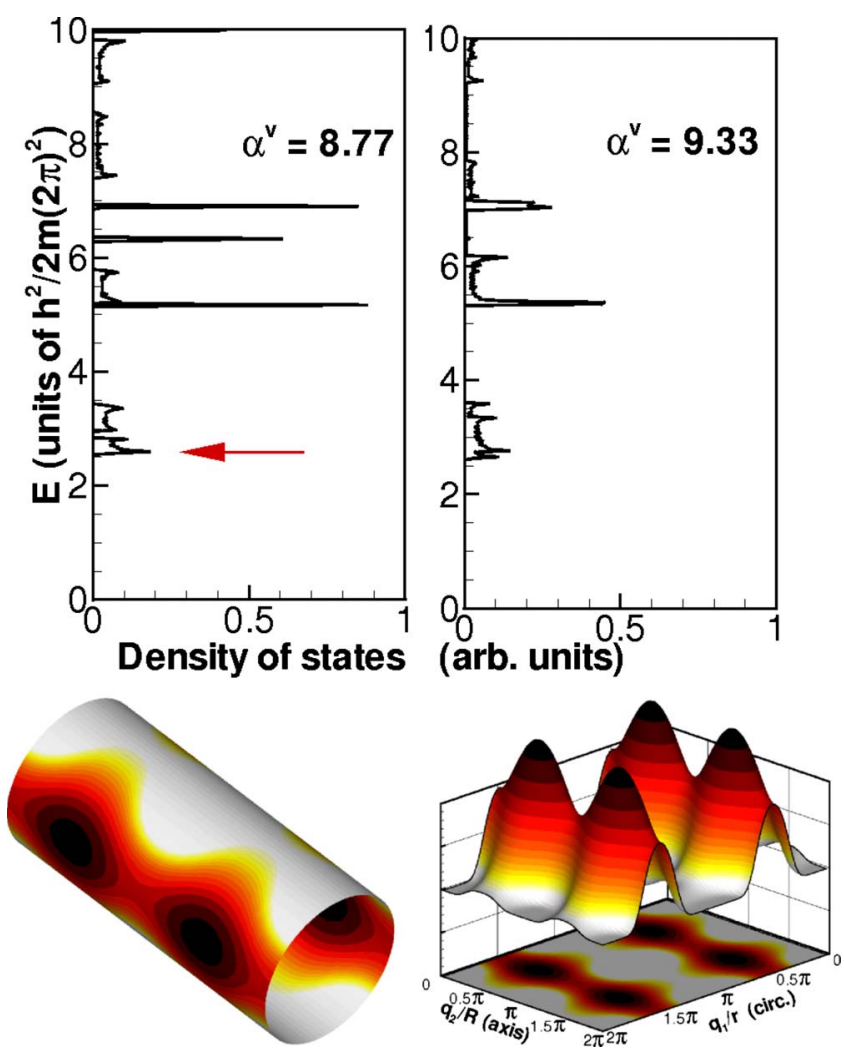

FIG. 11. (Color online) Top: DOS at $\rho=1.40$ for $\alpha^{v}=8.77$ and 9.33. Bottom: Probability density for a state belonging to the peak indicated with the arrow in the top panel. Darker color means higher density. In this case, tunneling between the dots is not negligible along the axis direction, while it is almost suppressed around the circumference.

with the arrow in the top panel. A rearrangement of the charge density in a lattice of quasi-0D regions is obtained analogously to the $\rho<1$ case. However, the unit cell $(\pi r$ $\times \pi R$ ) is now shorter along the cylinder axis, and tunneling between the quasi-0D states is larger along the axis direction. Therefore, the magnetic field induces a localization of the carriers in two arrays of tunnel-coupled dots along the axis direction. The two arrays on opposite sides of the cylinder are weakly coupled by tunneling. Also in this case, the localization is stronger for increasing $\alpha^{v}$ and does not depend on the sign of the charge or on the direction of the field. When the limiting case of $\rho$ much greater than 1 is approached, the tunneling between dots in the circumference direction is absent and the localized states result in two 1D-strips on the sides of the cylinder, which are not modulated along the axis.

\section{Dotlike localization}

The $\rho=1$ case is an admittedly difficult condition to be obtained exactly, but it is discussed here for completeness and as an example of the intermediate regime $r \approx R$. The energy bands are shown in the central panels of Fig. 7 . Again, for $\alpha^{v}=2.5$ a flat band is present and the width of this band is modulated by the magnetic field that affects tunneling. 


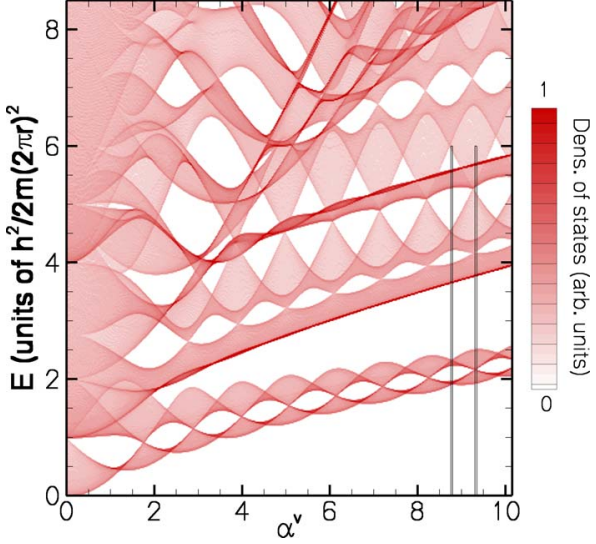

FIG. 12. (Color online) DOS at $\rho=1$ (see Sec. IV C) as a function of the energy and the coupling parameter $\alpha^{v}$ (color code given in the legend). The DOS shown in Fig. 13 corresponds to the two vertical lines at $\alpha^{v}=8.77$ and 9.33 .

The DOS is shown in Fig. 12 as a function of the parameter $\alpha^{v}$. Now, the modulation of the DOS shows regular patterns of energy gaps and peaks periodic with $\alpha^{v}$. A detailed analysis of these features will be given in Sec. IV D. The DOS at selected values of $\alpha^{v}$ is also shown in Fig. 13, where the peaks and gaps are clearly visible.

In the bottom panel of Fig. 13 we show the probability density on the tube for a state belonging to the peak indicated
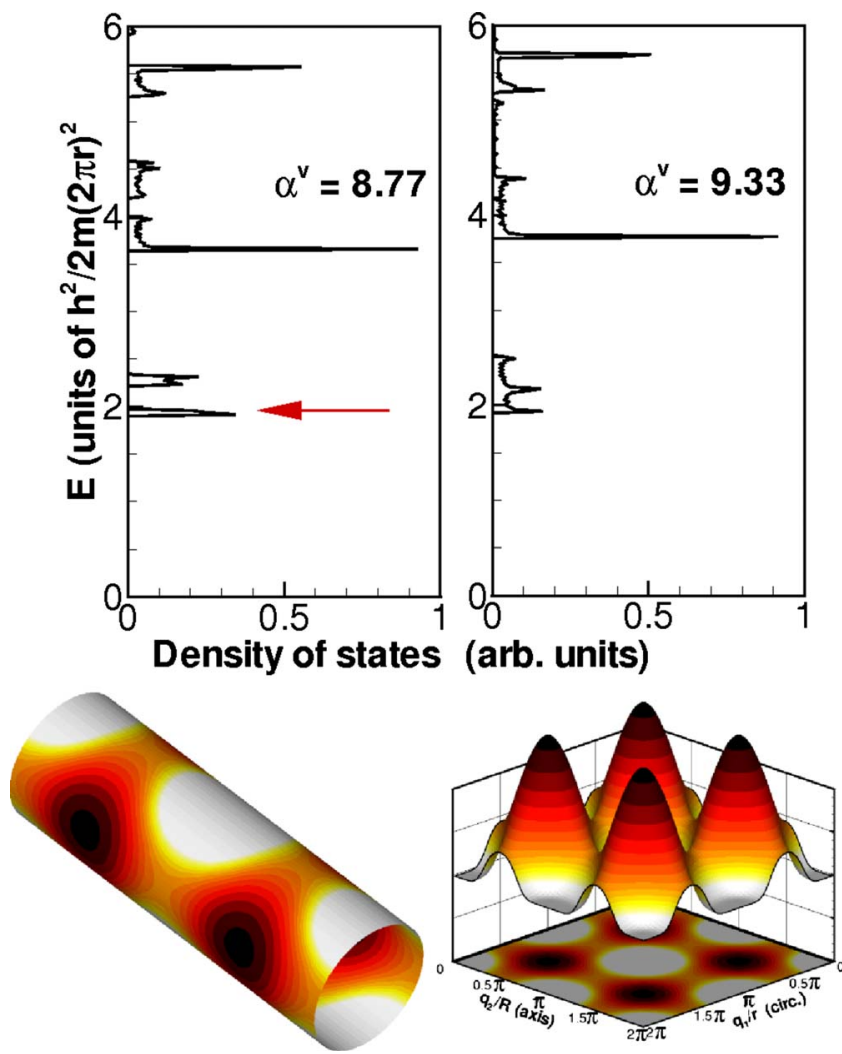

FIG. 13. (Color online) Top: DOS at $\rho=1$ for $\alpha^{v}=8.77$ and 9.33 . Bottom: Probability density for a state belonging to the peak indicated with the arrow in the top panel. Darker color means higher density. with the arrow in the top panel. Due to the additional symmetry of the $(\pi r \times \pi R)$ unit cell, the tunneling probability between the quasi-0D states is now the same along the axis and around the circumference directions, and a superlattice of dots each connected by four arms to neighboring dots is formed.

To summarize, we expect that at $r \approx R$ a periodically modulated magnetic field creates a square lattice of $0 D$ states. The localization is stronger for larger intensities and independent from the direction and the sign of the carriers. When $r$ substantially deviates from $R$, the tunnel coupling between the dots increases either along cylinder axis $(r$ $>R$ ), creating two weakly coupled 1D arrays of quantum dots, or along the cylinder circumference $(r<R)$, making the system more similar to a $1 \mathrm{D}$ array of weakly coupled quantum rings.

\section{Insights from the energy landscape}

Having established in Secs. I and III how the energy levels and the ensuing DOS are strongly affected by the intensity and modulation of the magnetic fields, we now look more closely to the overall behavior with respect to the interaction parameter $\alpha^{v}$. This will give us a deeper insight into the physics governing this system and exposes the analogies and consistencies with other physical situations.

\section{Aharonov-Bohm oscillations}

We first focus on the low-energy part of the spectrum and consider for definiteness the case $\rho=1$. A peculiar characteristic of the DOS (Fig. 12) is the oscillation of the energy levels so to form a plait. Figure 14 shows the energy levels at $k_{2}=0$ with the oscillatory behavior of the lowest levels with $\alpha^{v}$. This trend is a characteristic of many electronic systems under the effect of a magnetic field, and it is a typical fingerprint of Aharonov-Bohm-type behavior. ${ }^{38,42}$ In this specific device, the dots and the arms connecting them are regions of zero field that constitute a loop around a region of nonzero perpendicular component of the magnetic field, as shown in the bottom panel of Fig. 13. Clearly, this explains qualitatively the shift of the energy levels with $\alpha^{v}$, although our explicit calculation must take into account that the rings are not well defined for low values of $\alpha^{v}$, since the tunneling between the dots can be very high and the dots themselves are quite large. Furthermore, the direction and intensity of the field are not constant throughout the ring and, finally, the shape of the ring is not round but more squarelike. This explains why the minimum of the energy of the ground state is not constant against $\alpha^{v}$ as it would be in a textbook Aharonov-Bohm system. Furthermore the periodicity of the oscillations is not strictly an integer of the ratio $\Phi / \Phi_{0}$. An analogous behavior is revealed in self-assembled semiconductor rings, where the energy dependence on the magnetic field is explained taking into account the finite width of the ring and the nonuniform confining potential. ${ }^{43,44}$ Note that in this system the Aharonov-Bohm ring is not physically defined in the absence of the field and is induced by the same magnetic field and its interplay with the geometry of the C2DEG. 

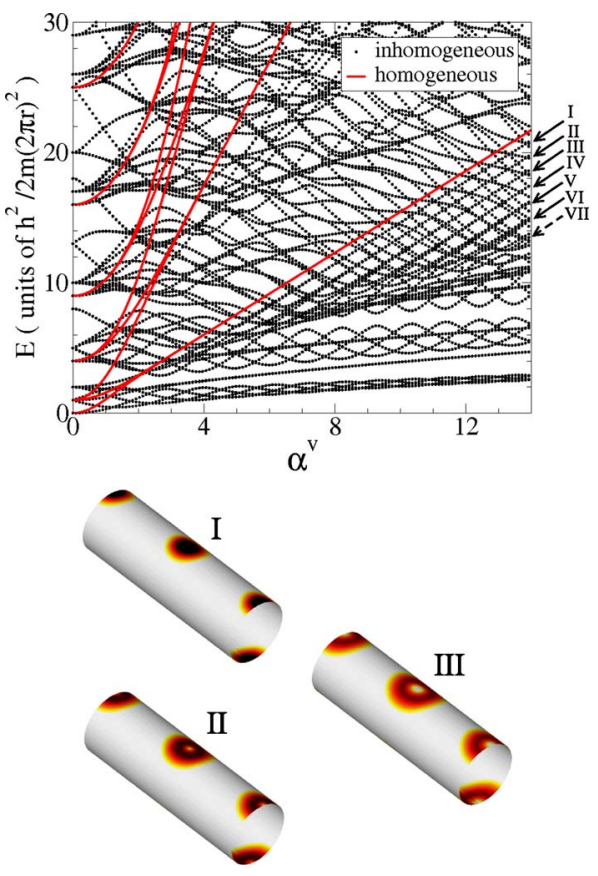

FIG. 14. (Color online) Top: the energy levels at $k_{2}=0$ are presented as a function of $\alpha^{v}$ in the case of $\rho=1$ up to the 100th level. The bands corresponding to the Landau levels are indicated by the arrows on the right axis. The Landau levels for the corresponding case with a homogeneous field are shown with a solid line. Bottom: The probability densities of the system for the Landau levels I, II, and III (see the arrows on the left plot) are shown. Darker color means higher density. The carriers are confined in the regions with the field perpendicular to the cylinder surface.

\section{Landau levels}

In Sec. III we have connected the energy levels at high homogeneous magnetic field with the formation of Landau levels on the surface of the tube. In the inhomogeneous case the lowest energy states are confined close to the regions where the field is parallel to the surface or its intensity goes to zero (we recall that the average field is zero in the present investigation). Since the Landau levels cannot appear in regions of zero perpendicular field, they are not involved in the formation of low-energy states. They form at higher energy instead. Figure 14 compares the $k_{2}=0$ energy levels of the homogeneous with inhomogeneous cases, for the same $r$, in a broad energy range (up to the 100th level). A set of levels, indicated by arrows and roman numerals, shows a linear shift with $\alpha^{v}$ with the same slope as the first Landau level of the homogeneous case. Plots of the carrier densities show that these states correspond to carriers confined in the region where the field is perpendicular to the cylinder surface, that is, the regions circulated by the Aharonov-Bohm-type rings which localize the low-energy states. These are highly degenerate states due to the flat dispersion with respect to $k_{y}$ and $n$. Clearly, the degeneracy is not the same as for genuine 2D Landau levels due to the periodicity imposed by the modulated field and the cylindrical symmetry. The degeneracy can also be traced to the charge density (Fig. 14, right panels), which is redistributed in dots that are well separated. Tunneling between these regions is completely suppressed.

\section{CONCLUSIONS}

We have investigated theoretically the effect of a magnetic field, either homogeneous or periodically modulated along the cylinder axis, on the electronic states in a C2DEG. The combined effects of the 2D-system curvature and the field can lead to carrier localization effects that are at the base of peculiar features of the DOS. In particular, we described how it is possible to alter the dimensionality of the states using the magnetic field.

As described in Sec. III, in a homogeneous magnetic field, the extended states of the cylinder form either a single or two 1D channels, depending on the field strength, localized in different regions of the cylinder. In the case of a field periodically modulated in space, the carriers are localized into a superlattice of tunnel-coupled quasi-0D states as we showed in Sec. IV.

The localization type (cylindrical 2D, 1D, and 0D) is only determined by the ratio between the diameter of the nanostructure, the magnetic length, and, possibly, the wavelength of the field modulation. Therefore a change in dimensionality of the carrier states can be achieved in any C2DEG at the appropriate field intensity. It turns out that for usual laboratory fields, in the few Tesla regimes, carrier localization occurs for C2DEGs with diameters in the few tens of nanometers range. This dimension is typical of semiconductor nanotubes, ${ }^{5-8,11}$ while smaller systems, as carbon nanotubes, would need much larger fields to reveal the same effects.

The carrier localization and DOS modulation should alter significantly the transport properties of the C2DEG. For example, the excitonic recombination efficiency is strongly dependent on the overlap and dimensionality of electron and hole states ${ }^{45-47}$ that, in turn, depends on the amplitude of the homogeneous magnetic field. We have also found clear signs of incipient Landau levels similar to the familiar 2DEG case and, in the modulated-field case, also Aharonov-Bohm type of oscillations in the low-energy spectrum.

\section{ACKNOWLEDGMENTS}

The authors are pleased to thank Giampaolo Cuoghi for the fruitful discussions. This work was partially supported by Project FIRB No. RBIN04EY74. 
*giulio.ferrari@unimore.it

${ }^{1}$ L. V. Radushkevich and V. M. Lukyanovich, Zh. Fiz. Khim. 26, 88 (1952).

${ }^{2}$ A. Oberlin, M. Endo, and T. Koyama, J. Cryst. Growth 32, 335 (1976).

${ }^{3}$ S. Iijima, Nature (London) 354, 56 (1991).

${ }^{4}$ M. S. Dresselhaus, P. Avouris, M. Endo, H. Dai, J.-C. Charlier, S. Iijima, R. Tenne, A. K. Zettl, S. G. Louie, Z. Yao, C. Dekker, T. Wang Odom, J. H. Hafner, C. M. Lieber, R. Saito, H. Kataura, J. H. Fink, P. Lambin, J. Hone, B. I. Yakobson, L. Forró, C. Schönenberger, P. M. Ajayan, and O. Z. Zhon, in Carbon Nanotubes: Synthesis, Structure, Properties, and Applications, edited by M. S. Dresselhaus, G. Dresselhaus, and P. Avouris (SpringerVerlag, Berlin, 2000)

${ }^{5}$ V. Y. Prinz, V. A. Seleznev, A. K. Gutakovsky, A. V. Chehovskiy, V. V. Preobrazhenskii, M. A. Putyato, and T. A. Gavrilova, Physica E (Amsterdam) 6, 828 (2000).

${ }^{6}$ O. G. Schmidt and K. Eberl, Nature (London) 410, 168 (2001).

${ }^{7}$ A. Lorke, S. Böhm, and W. Wegscheider, Superlattices Microstruct. 33, 347 (2003).

${ }^{8}$ N. Shaji, H. Qin, R. H. Blick, L. J. Klein, C. Deneke, and O. G. Schmidt, Appl. Phys. Lett. 90, 042101 (2007).

${ }^{9}$ J. Westwater, D. P. Gosain, and S. Usui, Phys. Status Solidi A 165, 37 (1998).

${ }^{10}$ T. Martensson, M. Borgström, W. Seifert, B. J. Ohlsson, and L. Samuelson, Nanotechnology 14, 1255 (2003).

${ }^{11}$ A. Fontcuberta i Morral, D. Spirkoska, J. Arbiol, M. Heigoldt, J. R. Morante, and G. Abstreiter, Small 4, 899 (2008).

${ }^{12}$ P. D. Ye, D. Weiss, R. R. Gerhardts, M. Seeger, K. von Klitzing, K. Eberl, and H. Nickel, Phys. Rev. Lett. 74, 3013 (1995).

${ }^{13}$ P. D. Ye, D. Weiss, R. R. Gerhardts, G. Lütjering, K. von Klitzing, and H. Nickel, Semicond. Sci. Technol. 11, 1613 (1996).

${ }^{14}$ D. Yoshioka and Y. Iye, J. Phys. Soc. Jpn. 56, 448 (1987).

${ }^{15}$ R. Yagi and Y. Iye, J. Phys. Soc. Jpn. 62, 1279 (1993).

${ }^{16}$ P. Vasilopoulos and F. M. Peeters, Superlattices Microstruct. 7, 393 (1990).

${ }^{17}$ F. M. Peeters and P. Vasilopoulos, Phys. Rev. B 47, 1466 (1993).

${ }^{18}$ I. S. Ibrahim and F. M. Peeters, Phys. Rev. B 52, 17321 (1995).

${ }^{19}$ D. R. Hofstadter, Phys. Rev. B 14, 2239 (1976).

${ }^{20}$ R. R. Gerhardts, D. Weiss, and K. v. Klitzing, Phys. Rev. Lett. 62, 1173 (1989).

${ }^{21}$ K. Ensslin and P. M. Petroff, Phys. Rev. B 41, 12307 (1990).

${ }^{22}$ B. S. DeWitt, Rev. Mod. Phys. 29, 377 (1957).

${ }^{23}$ H. Jensen and H. Koppe, Ann. Phys. (N.Y.) 63, 586 (1971).
${ }^{24}$ R. C. T. da Costa, Phys. Rev. A 23, 1982 (1981).

${ }^{25}$ M. Encinosa and L. Mott, Phys. Rev. A 68, 014102 (2003).

${ }^{26}$ A. Marchi, S. Reggiani, M. Rudan, and A. Bertoni, Phys. Rev. B 72, 035403 (2005).

${ }^{27}$ G. J. Meyer, N. L. Dias, R. H. Blick, and I. Knezevic, IEEE Trans. Nanotechnol. 6, 446 (2007).

${ }^{28}$ H. Taira and H. Shima, J. Phys.: Conf. Ser. 61, 1142 (2007).

${ }^{29}$ M. L. Leadbeater, C. L. Foden, J. H. Burroughes, M. Pepper, T. M. Burke, L. L. Wang, M. P. Grimshaw, and D. A. Ritchie, Phys. Rev. B 52, R8629 (1995).

${ }^{30}$ K.-J. Friedland, R. Hey, H. Kostial, A. Riedel, and K. H. Ploog, Phys. Rev. B 75, 045347 (2007).

${ }^{31}$ A. B. Vorob'ev, K.-J. Friedland, H. Kostial, R. Hey, U. Jahn, E. Wiebicke, J. S. Yukecheva, and V. Y. Prinz, Phys. Rev. B 75, 205309 (2007).

${ }^{32}$ G. Ferrari and G. Cuoghi, Phys. Rev. Lett. 100, 230403 (2008).

${ }^{33}$ H. Ajiki and T. Ando, J. Phys. Soc. Jpn. 62, 1255 (1993).

${ }^{34}$ E. Perfetto, J. Gonzàlez, F. Guinea, S. Bellucci, and P. Onorato, Phys. Rev. B 76, 125430 (2007).

${ }^{35}$ G. J. Meyer and I. Knezevic, J. Comput. Electron. 6, 219 (2007).

${ }^{36}$ R. E. Peierls, Z. Phys. 80, 763 (1933).

${ }^{37}$ V. Mitin, V. Kochelap, and M. Stroscio, Quantum Heterostructures (Cambridge University Press, Cambridge, England, 1999).

${ }^{38}$ S. Olariu and I. I. Popescu, Rev. Mod. Phys. 57, 339 (1985).

${ }^{39}$ H. Aoki and H. Suezawa, Phys. Rev. A 46, R1163 (1992).

${ }^{40}$ J. H. Kim, I. D. Vagner, and B. Sundaram, Phys. Rev. B 46, 9501 (1992).

${ }^{41}$ H. Ajiki and T. Ando, Physica B (Amsterdam) 201, 349 (1994).

${ }^{42}$ A. Fuhrer, S. Lüscher, T. Ihn, T. Heinzel, K. Ensslin, W. Wegscheider, and M. Bichler, Nature (London) 413, 822 (2001).

${ }^{43}$ N. A. J. M. Kleemans et al., Phys. Rev. Lett. 99, 146808 (2007).

${ }^{44}$ V. M. Fomin, V. N. Gladilin, S. N. Klimin, J. T. Devreese, N. A. J. M. Kleemans, and P. M. Koenraad, Phys. Rev. B 76, 235320 (2007).

${ }^{45}$ G. Bastard, E. E. Mendez, L. L. Chang, and L. Esaki, Phys. Rev. B 26, 1974 (1982).

${ }^{46}$ F. Rossi and E. Molinari, Phys. Rev. Lett. 76, 3642 (1996).

${ }^{47}$ F. Rossi, G. Goldoni, and E. Molinari, Phys. Rev. Lett. 78, 3527 (1997).

${ }^{48}$ Here and in the following, each state $\varphi$ will be labeled with the quantum number $n$ of the zero-field state where it develops continuously as $B$ increases. This unambiguous correspondence is possible since the position of the zero-probability nodes of $\varphi$ is not altered by the magnetic field. 